$63^{\text {ème }}$ Congrès de la SFCO, 02003 (2015)

DOI:10.1051/sfco/20156302003

(C) Owned by the authors, published by EDP Sciences, 2015

\title{
COMMUNICATION
}

\section{Les canines incluses qui prennent leur temps...}

\author{
Benhamou Giuly A*, Rotenberg M** \\ * Interne en orthodontie DES3, UFR Odontologie, Toulouse \\ ** MCU-PH, UFR Odontologie, Toulouse
}

Peut-on établir une corrélation entre la position initiale d'une canine incluse maxillaire et la durée du traitement pour sa mise en place sur l'arcade ? L'âge et le sexe du patient ont-ils une influence ?

C'est la question posée au travers d'une étude observationnelle longitudinale prospective multicentrique menée pendant 15 mois.

L'étude s'intéresse à la prévalence de la population concernée (sexe, âge), à la répartition des canines (13, 23, 13 et 23), à leur maturité radiculaire, à leur position initiale ainsi qu'à leur durée de traitement. Les patients sont répertoriés par l'intermédiaire de plusieurs chirurgiens réalisant l'acte d'exposition chirurgicale. L'orthodontiste correspondant est alors contacté pour recueillir les différentes informations. Ainsi, plus de 100 patients en cours de traitement orthodontique et présentant au moins une canine incluse maxillaire ont été intégrés.

La position initiale de la canine est définie sur un orthopantomogramme ou un cone beam selon son inclinaison (verticale, oblique, horizontale) par rapport à la verticale, l'angle formé avec l'axe de l'incisive latérale, l'angle formé avec l'axe de la première prémolaire et selon la distance perpendiculaire de la pointe cuspidienne au plan d'occlusion. Leur position vestibulaire, palatine ou intermédiaire est aussi prise en compte.

La durée de l'étude s'étend du jour de l'exposition chirurgicale de la canine à la prise en charge de cette dent dans un arc sans contrainte.

L'analyse de la littérature sur les 10 dernières années n'évoque pas d'étude prospective c'est pourquoi le choix de ce type d'étude a été réalisé pour limiter les biais. Les résultats obtenus dans les différents articles montrent une corrélation entre la durée de traitement, l'âge et la situation de la canine incluse. Aucun dimorphisme sexuel n'a pu être mis en évidence.

Les buts de cette étude prospective sont d'une part de vérifier les résultats précédents et d'autre part de préciser au mieux la différence de durée de traitement selon la localisation initiale de la canine afin d'en informer le patient.

\section{Nom et adresse du conférencier}

\section{Audrey BENHAMOU GIULY}

Interne en orthodontie DES3

33 rue Raymond IV

31000 Toulouse (France)

audrey.benhamou@gmail.com

This is an Open Access article distributed under the terms of the Creative Commons Attribution License 4.0, which permits unrestricted use, distribution, and reproduction in any medium, provided the original work is properly cited. 